\title{
GRAIN FORMATION IN THE WINDS OF COOL RED GIANT STARS
}

\author{
E. SEDLMAYR AND J. M. WINTERS \\ Institut für Astronomie und Astrophysik \\ Technische Universität, Berlin, Germany
}

\begin{abstract}
The problem of dust formation in the circumstellar envelopes of Asymptotic Giant Branch stars is reviewed. Special emphasis is put on the consistent modelling of the dust-forming circumstellar shell, where due to a strong coupling the dust formation process governs the dynamical behavior of the object.
\end{abstract}

\section{Introduction}

Cosmic dust plays an important role in current astrophysics, manifesting itself as an important component of the interstellar medium and dominating the appearance of various objects like red giants and supergiants, and even episodic events such as R CrB stars, novae, and supernovae. For this reason it seems natural that cosmic grain physics, in particular the problem of grain formation, has attracted much interest in recent decades. In particular, red giants and supergiants, showing pronounced stellar outflows, have proven to be ideal objects to study in detail the process of grain nucleation and growth.

From a microscopic point of view, grain formation can be conceived as a sequence of chemical reactions, starting with suitable molecules and finally ending with macroscopic dust particles. In this respect, the grain formation process itself seems to be controlled only by proper pressure and temperature conditions and a certain chemical composition of the gas phase providing suitable molecules to condense. So far the problem appears to be rather simple: Adopting some nucleation theory, one has first to determine regions of high nucleation efficiency in the $p-T$ plane (i.e. regions of sufficiently high particle densities and sufficiently low temperatures), and second to determine those trajectories along the evolution of a material 
element which cross the dustforming region for a time sufficiently long to enable effective grain formation in the element. Natural systems for this process are stellar outflows, starting from the initial plasma phase in the stellar photosphere. With decreasing temperature a complex chemistry evolves and finally, at some critical radius, favorable conditions for dust nucleation and growth occur as indicated by the observed steep increase in the optical depth, the high infrared excess and a significant increase of the expansion velocity in the shells of these objects (cf. Figure 1). In this respect, dust formation in the winds of red giant stars is a physically and mathematically well posed problem, the initial conditions of which can be uniquely inferred from the atmospheric analysis.

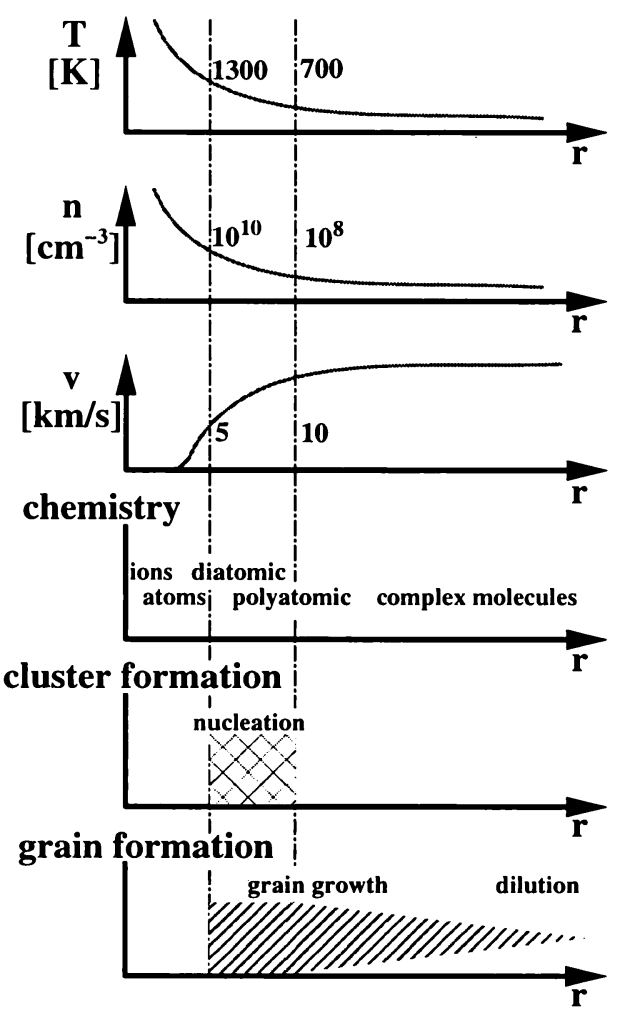

Figure 1. Schematic structure of a dust-forming circumstellar shell. Dust nucleation is well confined to a region approximately between $1300 \mathrm{~K}$ and $700 \mathrm{~K}$ while the region of dust growth extends much farther outward.

Despite this simple basic picture, astrophysical dust formation appears to be an extremely complicated non-linear process. The reason for this behavior is that the presence of even a small amount of dust, due to its huge absorption and scattering coefficient, has severe consequences for the local physical and chemical conditions in the dust-forming region. In particular the following three effects play an important role:

- Dust is a very effective transmitter of energy by efficiently absorbing and thermalizing short-wavelength photons and emitting their energy at infrared wavelengths. By this process, dust strongly affects the thermodynamical behavior of the system.

- Dust is a very effective transmitter of momentum by transferring the absorbed photon momentum by frictional coupling to the ambient gas. Therefore, dust strongly affects the hydrodynamical structure of the system. 
- Dust is a catalyst for chemical reactions: gas particles absorbed at the grain's surface can react with the grain material or another admolecule to form new species which cannot be formed from the gaseous phase. Hence dust strongly determines the chemical behavior of the system.

As a consequence, any realistic treatment of circumstellar dust formation can only be performed by means of a self-consistent modelling of the dustforming objects, with thermodynamics, chemistry, cluster physics, radiation/matter interaction, and nonlinear dynamics as essential ingredients (e.g. Sedlmayr \& Winters 1991; Fleischer et al. 1992; Winters et al. 1994a).

\section{Circumstellar Dust Formation}

The consistent treatment of a dust-forming shell of a red giant requires the consideration of at least three closely coupled complexes: circumstellar chemistry, the dust nucleation and grain growth problem, and the hydrodynamical and thermodynamical wind structure.

\subsection{CIRCUMSTELLAR CHEMISTRY}

In principle, chemistry plays a three-fold role with regard to circumstellar shells:

i) for the interpretation of the observed molecular lines, i.e. the diagnostics of the objects, the detailed chemical structure and thus the local concentrations of the molecular species have to be known,

ii) the chemical species present determine the transport coefficients in the atmosphere and hence the local physics in the inner parts of the shells (e.g. temperature, density, ...), and

iii) the chemistry determines in particular the local abundance of the dust-forming species and, thereby, the primary (high--temperature) condensates to be expected.

To give a general picture of the complex processes to be involved in modelling the chemistry of the circumstellar shell, Figure 2 is a sketch of the various chemical processes which might be important.

\subsubsection{Chemical equilibrium (CE)}

This most simple approach requires the chemical reaction timescales to be small compared to all other timescales determining the structure of the outflow (e.g. the hydrodynamical timescale). This condition only holds for sufficiently high particle densities, small velocity fields, and systems where photoreactions can be neglected, i.e. for radiation fields with a negligible UV component. This situation seems to be realized for the cool photospheres and inner shell regions of cool C stars and M stars without chromospheres. This CE condition is very severe and easily violated by the presence of even 


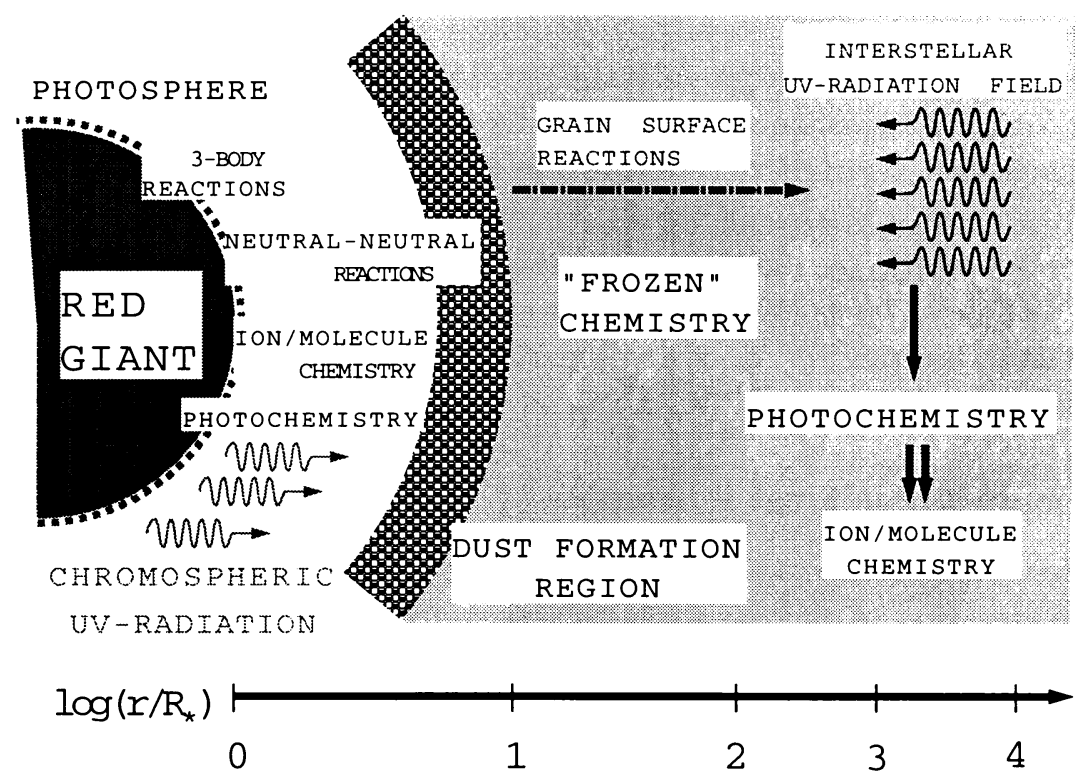

Figure 2. Chemical processes in different regions of the circumstellar shells around red giants (from Patzer 1998).

weak UV contributions and/or by moderate velocity fields (e.g. Goeres et al. 1988; Beck et al. 1992). Their model calculations for $\alpha$ Ori indicate dramatic deviations of particular species from CE caused by only a very moderate photospheric UV field $(T=3000 \mathrm{~K})$ or by small temporal variations of the radiation field as observed for $\alpha$ Ori (Dupree et al. 1987).

\subsubsection{Chemical non-equilibrium}

This situation requires the solution of the time-dependent network of chemical rate equations (non-kinetic equilibrium) in the case that velocity fields and/or short time variations of the radiation field have to be considered. This is the usual case for the modelling of long-period variable stars (LPVs), where the dynamical timescales determine the local physics. Such a treatment is also necessary in the outer regions of stationary outflows, where the hydrodynamical expansion and acceleration timescale becomes comparable to or even shorter than the chemical timescales involved. For these systems often a frozen chemistry evolves due to the rapid decrease of the chemical reaction rates caused by dilution in the wind. As an example, Figure 3 shows a comparison between a pure $\mathrm{CE}$ and a non-CE calculation of the chemical structure for a stationary dust-driven wind model describing the dust shell of a C star. 


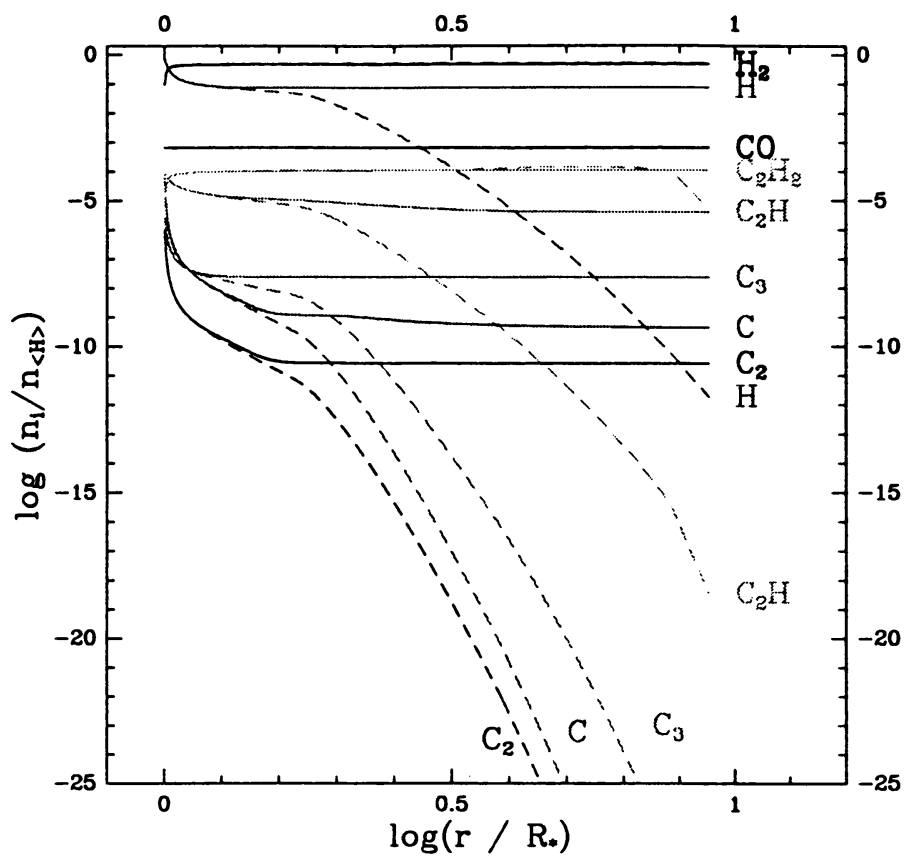

Figure 3. Equilibrium concentrations (dashed lines) and non-equilibrium concentrations (solid lines) for different molecules in a stationary model for a dust-driven wind. Due to fast dilution in the wind, a frozen chemistry develops in the non-CE calculation. The figure is from Patzer (1998).

\subsection{DUST NUCLEATION AND GROWTH}

Basically, dust formation can be thought of as a chain of chemical reactions starting with small molecules and ending with macroscopic specimens called dust grains. To follow this idea by a theoretical description requires, in principle, the construction and solution of a suitable chemical network, containing in the small regime molecules of increasing size, in the intermediate regime clusters (containing $\sim 10$ constituents) and in the large-particle regime grains having $\sim 10^{10}$ constituents. This approach, however, is much too elaborate not only due to the huge number of reaction equations (corresponding to the number of constituents), but also due to the lack of input data necessary to describe the reaction rates, structure, and thermodynamical properties of the particles involved. When applied to PAH formation in C-star outflows, this method turned out to be limited in practice to sizes of polyaromatic molecules having less than six aromatic rings (e.g. Cherchneff et al. 1992).

A detailed inspection of the dust formation process shows, however, that 
the problem can be split into two different phases which can be treated separately: $(i)$ the nucleation process, i.e. the formation of so-called critical clusters out of the gas phase, and ( $i i)$ the growth of these critical clusters to macroscopic specimens. As all clusters smaller than the critical cluster have the tendency to evaporate and all clusters larger than the critical cluster tend to grow, the critical cluster plays the role of a 'bottleneck,' which any effective dust formation process has to surmount in order to arrive at macroscopic grains. The properties of the critical cluster, defined by an extremum of its Gibbs free enthalpy, are therefore decisive for the whole dust formation problem and its efficiency.

\subsubsection{Nucleation regime}

For the treatment of the first phase - the formation of the critical cluster - usually two different methods are adopted:

1) Classical nucleation theory. In this concept, the molecule-cluster transition is conceived as a thermodynamic phase transition governed by the thermodynamic functions of the emerging products and by the supersaturation ratio of the condensing gaseous species. Classical nucleation theory results in a critical cluster having maximum free enthalpy and in a critical nucleation rate, solely given by the thermodynamic properties of the critical cluster and the local supersaturation ratio of the condensing species which has to be larger than unity to allow the formation of the seed particle. This method for example yields excellent results if applied to the formation of water droplets in cloud chamber experiments or in the earth's atmosphere but is highly questionable in application to astrophysical problems. This is due to the fact that classical nucleation theory conceptually refers to critical clusters having already well defined surfaces (which is true for water droplets consisting of at least $10^{9}$ monomers) but not for astrophysical critical clusters usually having about 5-20 basic constituents. In this case, the surface tension concept cannot be applied and the thermodynamic functions of all individual small clusters involved either have to be provided by suitable laboratory experiments or calculated by molecular dynamics methods (e.g. Patzer et al. 1995, Köhler et al. 1997). So far, however, such an approach has been limited to chemically homogeneous clusters, whose composition and the corresponding molecular interaction potentials are known. A similar description of heterogeneous structures is still lacking. For this reason, all nucleation theories applied so far in astrophysics consider the formation of homogeneous critical clusters (Gail \& Sedlmayr 1986; Kozasa \& Hasegawa 1987; Kozasa et al. 1996; Köhler et al. 1997).

2) Chemical pathway. This approach has been applied to the formation of polyaromatic molecules in C stars (e.g. Keller 1987; Cherchneff et al. 
1992; Goeres 1993; Goeres et al. 1996), polyaromatic carbons in R CrB stars (Goeres \& Sedlmayr 1992), and fullerenes in the Krätschmer-Huffman experiment (Krätschmer et al. 1990; Goeres \& Sedlmayr 1991). The basic idea of this method is that in the corresponding reaction networks a few dominant reaction pathways can always be isolated along which the grain formation process essentially proceeds. By a detailed discussion of the individual reactions a most efficient pathway can be constructed leading from the basic molecule (the monomer) into the regime beyond the critical cluster. On this path the critical cluster is defined as that step having the minimum net growth rate. Application of this method to PAH formation in $\mathrm{C}$-star winds shows that the critical cluster is rather small at low temperatures (less than $20 \mathrm{C}$ atoms for $T<1000 \mathrm{~K}$; see Goeres et al. 1996), and therefore only few reaction steps have to be considered.

\subsubsection{Growth regime}

This particle size regime, which is essentially defined by the addition of growth species to the already existing clusters for both the classical nucleation theory and the pathway description, can be treated with sufficient accuracy by assuming thermal growth (Gail \& Sedlmayr 1988; Gauger et al. 1990). This requires the knowledge of the corresponding reaction rates (i.e. the sticking coefficients) which in particular for inorganic grains are only poorly known or even completely lacking (e.g. Patzer et al. 1995).

\section{Models of Dust-Forming Circumstellar Shells}

In the context of circumstellar dust formation, three situations in particular have been modelled: $(i)$ stationary dust-driven winds, $(i i)$ shells of pulsating variables like LPVs, and ( $i i i)$ episodic phenomena including $\mathrm{R}$ CrB stars, novae and supernovae.

\subsection{STATIONARY DUST-DRIVEN WINDS}

This phenomenon, which only occurs for objects showing negligible variability and extremely high mass-loss rates, is confined to the upper-right region of the HR diagram (cf. Dominik et al. 1990). The physical condition for a stationary dust-driven wind to exist is that the dust formation region is situated inside the sonic point of the wind in order for the radiation pressure on dust to be able to levitate the atmosphere beyond the escape point and to determine the mass-loss rate of the star (Gail \& Sedlmayr 1987; Sedlmayr \& Dominik 1995). A good example for such an object is IRC +10216, the self-consistent model of which (assuming spherical symmetry, radiative equilibrium and chemical equilibrium, and applying classical nucleation theory to describe the formation of amorphous carbon grains) 
provides not only the observed velocity field, the temperature structure, and the optical depths, but also the observed spectral appearance and the detailed shell structure leading to the observed fringe visibilities (Winters et al. 1994a).

\subsection{PULSATING VARIABLES}

Most red giants surrounded by a circumstellar dust shell are long-period variables (e.g. Jura 1986) with pulsation periods on the order of $P \approx 1 \mathrm{yr}$. Modelling their circumstellar dust shells therefore requires a fully timedependent treatment of the problem. The results of such calculations reveal a very complex structure of the circumstellar shell (e.g. a layered distribution of the dust component, dust-induced or dust-accelerated shocks) and the existence of an eigenperiod of the circumstellar shell leading to the effect of multiperiodicity which is caused by the very strong and highly non-linear coupling among hydrodynamics, thermodynamics, chemistry, and dust formation and growth (see Fleischer et al. 1991, 1992, 1995, 2000). These phenomena provide a physical explanation for the internal structures and the multiperiodic long-term behavior of the observed infrared lightcurves (e.g. Feast et al. 1984; Le Bertre 1992) and of the observed brightness profiles and corresponding fringe visibilities (e.g. Ridgway \& Keady 1988; Danchi et al. 1994) of these objects as discussed in Winters et al. (1994b, 1995).

\subsection{DUST FORMATION IN R CrB STARS}

A third important astrophysical application of dust formation theories concerns the study of episodic phenomena like nova and supernova outflows and the $\mathrm{R} \mathrm{CrB}$ occultation events. Whereas the first two cases can be treated simply by adopting a stationary flow, dust formation in $\mathrm{R} \mathrm{CrB}$ environments turns out to be triggered by a critical phenomenon causing an instantaneous phase transition from molecules to carbon dust (cf. Goeres \& Sedlmayr 1992). As R CrB even in the quiet state shows more or less periodic variations, the trigger mechanism might be some critical shock wave causing an extreme local increase in the temperature (up to $\sim 50000 \mathrm{~K}$ ), thereby providing an excellent initial condition for a subsequent cooling flow down to well below the radiative equilibrium temperature within only a few days (Woitke et al. 1996). This idea appears to be very appealing because it not only explains the observed large optical depths causing the obscuration but even seems to solve the long-standing debate between observers and theoreticians concerning the expected site of dust formation. Previous theories always claimed that dust formation takes place at a distance of about 10 stellar radii, where the temperature is low enough for 
grains to be able to form. This contradicts the observations which can only be explained if dust formation takes place very close to the star. This discrepancy could be resolved by the mechanism suggested above as it yields extreme supercooling and therefore high nucleation rates even at about 2 stellar radii (Woitke et al. 1996). However, a consistent modelling of this mechanism would require a 3-dimensional treatment of cloud formation which seems to be out of the scope of present-day capabilities. Moreover, atmospheres of R CrB stars seem to be close to their Eddington limit (Asplund 2000), suggesting sporadic "blob" outflows of matter which also could provide favorable conditions for dust formation along their cooling tracks.

\section{Conclusions}

The consistent modelling of red giants and supergiants, novae, and supernovae, including chemistry and dust formation, and the investigation of the obscuration phenomenon of $\mathrm{R}$ CrB stars, all yield results in excellent agreement with the observed spectral appearance and the timescales characterizing these objects. Nevertheless, all theories applied so far still suffer from rather restrictive assumptions either with regard to the microscopic physics (e.g. chemical equilibrium, classical nucleation theory, ...) or in order to reduce the numerical effort (e.g. spherical symmetry, ... ). These limitations should be surmounted in future work in order to arrive at a realistic, consistent description and understanding of these interesting objects. This need is especially urgent in view of already existing and future high-precision instruments, which are collecting a wealth of very detailed observations, waiting to be interpreted on an equivalently high level.

We thank Beate Patzer and Axel Fleischer for useful comments on the manuscript. This work was supported by the BMBF (grant 05 3BT13A 6) and by the DFG (grant Se 420/8-1).

\section{References}

Asplund, M. 2000, in IAU Symp. 177: The Carbon Star Phenomenon, ed. R.F. Wing (Kluwer), p. 521

Beck, H. K. B., Gail, H.-P., Henkel, R. \& Sedlmayr, E. 1992, A\&A, 265, 626

Cherchneff, I., Barker, J. R. \& Tielens, A. G. G. M. 1992, ApJ, 401, 269

Danchi, W. C., Bester, M., Degiacomi, C. G., Greenhill, L. J. \& Townes, C. H. 1994, AJ, 107,1469

Dominik, C., Gail, H.-P., Sedlmayr, E. \& Winters, J. M. 1990, $A \& A, 240,365$

Dupree, A. K., Baliunas, S. L., Guinan, E. F., Hartmann, L., Nassiopoulos, G. E. \& Sonneborn, G. 1987, $A p J, 317$, L85

Feast, M. W., Whitelock, P.A., Catchpole, R. M., Roberts, G. \& Overbeek, M. D. 1984, $M N R A S, 211,331$

Fleischer, A. J., Gauger, A. \& Sedlmayr, E. 1991, $A \& A, 242, \mathrm{~L} 1$

Fleischer, A. J., Gauger, A. \& Sedlmayr, E. 1992, $A \& A, 266,321$ 
Fleischer, A. J., Gauger, A. \& Sedlmayr, E. 1995, $A \& A A, 297,543$

Fleischer, A. J., Winters, J. M. \& Sedlmayr, E. 2000, in IAU Symp. 177: The Carbon Star Phenomenon, ed. R. F. Wing (Kluwer), p. 377

Gail, H.-P. \& Sedlmayr, E. 1986, $A \& A, 166,225$

Gail, H.-P. \& Sedlmayr, E. 1987, $A \& A, 177,186$

Gail, H.-P. \& Sedlmayr, E. 1988, $A \& A, 206,153$

Gauger, A., Gail, H.-P. \& Sedlmayr, E. 1990, $A \& A, 235,345$

Goeres, A. 1993, Rev. Mod. Astron., 6, 165

Goeres, A., Henkel, R., Sedlmayr, E. \& Gail, H.-P. 1988, Rev. Mod. Astron., 1, 231

Goeres, A., Keller, R., Sedlmayr, E. \& Gail, H.-P. 1996, Polycyclic Aromatic Compounds, 8,129

Goeres, A. \& Sedlmayr, E. 1991, Chem. Phys. Lett., 184, 310

Goeres, A. \& Sedlmayr, E. 1992, $A \& A, 265,216$

Jura, M. 1986, ApJ, 309, 732

Keller, R. 1987, in Polycyclic Aromatic Hydrocarbons and Astrophysics, ed. A. Léger, L. d'Hendecourt and N. Boccara (Reidel), p. 387

Köhler, T. M., Gail, H.-P. \& Sedlmayr, E. 1997, A\&A, 320, 553

Kozasa, T., Dorschner, J., Henning, T. \& Stognienko, R. 1996, A\&A, 307, 551

Kozasa, T. \& Hasegawa, H. 1987, Prog. Theor. Phys., 77, 1402

Krätschmer, W., Fostiropoulos, K. \& Huffman, D. R. 1990, Chem. Phys. Lett., 170, 167

Le Bertre, T. 1992, A\&A Supp., 94, 377

Patzer, B. 1998, Ph.D. Dissertation, Technische Universtät, Berlin, Germany

Patzer, A. B. C., Köhler, T. M. \& Sedlmayr, E. 1995, Planet. \& Space Sci., 43, 1233

Ridgway, S. T. \& Keady, J. J. 1988, ApJ, 326, 843

Sedlmayr, E. \& Dominik, C. 1995, Space Sci. Rev., 73, 211

Sedlmayr, E. \& Winters, J. M. 1991, in Stellar Atmospheres: Beyond Classical Models, ed. L. Crivellari, I. Hubeny and D. G. Hummer (Kluwer), p. 397

Winters, J. M., Dominik, C. \& Sedlmayr, E. 1994a, $A \& A, 288,255$

Winters, J. M., Fleischer, A. J., Gauger, A. \& Sedlmayr, E. 1994b, $A \& A, 290,623$

Winters, J. M., Fleischer, A. J., Gauger, A. \& Sedlmayr, E. 1995, $A \& A$ A, 302, 483

Woitke, P., Goeres, A. \& Sedlmayr, E. 1996, A\&sA, 313, 217

\section{Discussion}

Linsky: Could you mention what critical laboratory or theoretical crosssections and rates are needed to make major progress on this topic?

Sedlmayr: There is generally a need for cross-sections and thermodynamic data, in particular for calculating the silicon and sulfur chemistry in circumstellar envelopes. Also many neutral-neutral and three-body reactions -- necessary for SiC-nucleation, for example are unknown. Of great interest also are unimolecular reactions, like carbon chain to ring transitions, which play a role in the carbon grain formation in $\mathrm{R}$ CrB atmospheres.

Gustafsson: I do agree with your view that one should strive for detailed self-consistent theoretical modelling and avoid semi-empirical models with dubious or even meaningless free fitting parameters. However, observations now accumulate indicating the presence of inhomogeneities in circumstellar envelopes, and self-consistent models of these are, probably still far off. For 
these phenomena we need to develop a physically reasonably sound semiempirical approach.

Sedlmayr: This is just the point. On the one hand we have a great many excellent observations revealing both the overall appearance and also the whole variety of details. On the other hand we have the theoretical description based on our incomplete understanding of the relevant physical processes. The only way to connect the observations and theory is to develop consistent theoretical (yet highly simplified) models and hope to theoretically reproduce the structures observed. In this way an iteration procedure between theory and observation is defined which finally should lead to a real physical description and understanding of the observed phenomena. 